\title{
Non-invasive detection of matrix-producing chondrocytes in tissue-engineered cartilage by second-harmonic-generation microscopy
}

\author{
Reiko MAEHARA*, Shuichiro FUKUSHIMA*, Masahiro KINO-OKA** and Tsutomu ARAKI* \\ * Department of Mechanical Science and Bioengineering, Osaka University, \\ 1-3 Machikaneyama, Toyonaka, Japan \\ E-mail: maehara@sml.me.es.osaka-u.ac.jp \\ ** Department of Biotechnology, Osaka University, \\ 2-1 Yamadaoka, Suita, Japan
}

Received 21 July 2013

\begin{abstract}
Noninvasive quality evaluation methods that make it possible to monitor culture processes are ideal for tissue-engineered cartilage made of autologous chondrocytes and collagen gel. However, usual methods are quite invasive because the products are examined chemically and observed histologically with dye. Here we employed second-harmonic-generation (SHG) microscopy, which can be used to observe collagen without any preparation such as staining or fixing. We demonstrated SHG imaging of tissue-engineered cartilage composed of rabbit chondrocytes and type I collagen gel. Our results showed that aggregations of round cells that produced type II collagen were observed as spherical dark spaces in the SHG images. The volumes of these spaces were related to the numbers of cells in the aggregations. These results indicate that we can estimate the number of the matrix-producing cells by SHG imaging. Because SHG microscopy is a noninvasive method, it is suitable for evaluating the quality of tissue-engineered cartilage and also for quality control during the manufacturing process.
\end{abstract}

Keywords : Collagen, Nonlinear optical microscopy, Regenerative medicine

\section{Introduction}

In current regenerative medicine for treating knee joints, tissue-engineered cartilage is transplanted into the joints. For culturing chondrocytes for use in this treatment, collagen is a popular scaffold material due to its biocompatibility (Ahmed and Hincke, 2010). For example, Ochi et al. transplanted autologous chondrocytes cultured in atelocollagen gel into a human knee joint and reported that the transplantation promoted restoration of the articular cartilage (Ochi, et al., 2002). Although they performed sterility testing repeatedly during the culture period by checking for bacterial growth in the culture medium on blood agar plates, the quality of the tissue-engineered cartilage that was transplanted into the patient's body was not evaluated. In our opinion, quality evaluation of tissue-engineered cartilage must be carried out before clinical use. However, existing evaluation techniques involving cell counting require physically and/or chemically invasive treatment, such as staining, sectioning, and decomposing. Another problem with these treatments is that they result in lower cell fabrication efficiency because the examined cells cannot be used for transplantation. Therefore, extra cartilage has to be prepared for the evaluation. The lower cell fabrication efficiency is a critical problem because chondrocytes dedifferentiate when they are cultured in vitro, and only primary cells can be applied to make tissue-engineered cartilage. Consequently, there is a strong demand for a noninvasive method of evaluating tissue-engineered cartilage that does not reduce the number of matrix-producing chondrocytes that can be used for transplantation.

Among the various noninvasive tissue observation techniques that are available, second-harmonic-generation 
(SHG) microscopy is the most attractive for visualizing fibrous collagen (Freund, et al., 1986). Because SHG is an intrinsic phenomenon occurring in the microstructure of the collagen, treatments such as fixation and staining are not required for observing the collagen. Among the various materials used for scaffolding in chondrocyte culturing, type I collagen is dominantly used for culturing of tissue-engineered cartilage. This collagen emits strong SHG light because the molecules join together and construct a hierarchical fibrous structure that results in the amplification of SHG light. Therefore, SHG microscopy can provide us with morphological information about chondrocyte culturing via the collagen molecule.

In the present research, we observed remodeling of collagen during the culturing of chondrocytes in collagen gel and found a characteristic SHG profile of matrix-producing cells. The SHG profile enabled us to estimate the distribution of intact cells that produced the cartilaginous matrix. Based on these findings, we propose a possible method for evaluating the quality of tissue-engineered cartilage.

\section{Materials and Methods 2.1 Cell Culture}

Chondrocytes were isolated from the articular cartilage extracted from a Japanese white rabbit according to the procedure of Yamamoto et al. (2002) and were cryopreserved at $-196{ }^{\circ} \mathrm{C}$. The cells were thawed and suspended in culture medium, and mixed with fourfold volume of 3\% type I collagen solution (Atelocollagen implant, Koken Co., Tokyo, Japan). The mixture was placed in 3 culture dishes $(100 \mu \mathrm{L} / \mathrm{dish})$. The final cell seeding densities in collagen gel for high and low seeding density group were $2.0 \times 10^{5}$ and $2.0 \times 10^{6}$ cells $/ \mathrm{mL}$, respectively. According to previous research, the cell number of both cultures slightly decreased in 7 days after seeding, and increased logarithmically after that (Yamamoto, et al., 2002). Khoshfetrat et al. (2009) reported that the low-density seeding induced dedifferentiation of chondrocytes, whilst cells seeded at high density grew well with chondrocyte phenotypes. The dishes were filled with Dulbecco's modified Eagle's medium (Sigma-Aldrich, St. Louis, MO, USA) supplemented with $10 \%$ fetal bovine serum, antibiotics (100 units $/ \mathrm{mL}$ penicillin $\mathrm{G}, 0.1 \mathrm{mg} / \mathrm{mL}$ streptomycin, and $0.25 \mathrm{mg} / \mathrm{mL}$ amphotericin B, all obtained from Gibco, Invitrogen, Life Technologies Co., Carlsbad, CA, USA), and 0.17 $\mu \mathrm{mol} / \mathrm{mL}$ L-ascorbic acid (Wako Pure Chemical Industries, Osaka, Japan), and then incubated at $37{ }^{\circ} \mathrm{C}$ under an atmosphere of $5 \% \mathrm{CO}_{2}$.

\subsection{Sample Preparation}

Tissue-engineered cartilage was harvested from the culture dishes at 3, 8, and 15 days after seeding and was then cut into eight pieces with a surgical knife. For observation of the cell morphology and distribution, cytoplasm was labeled with calcein AM fluorochrome (Molecular Probes, Life Technologies Co., Carlsbad, CA, USA), diluted with Dulbecco's phosphate buffered saline (D-PBS) according to the manufacturer's standard procedure. The specimens were then fixed with 4\% paraformaldehyde. Type II collagen, which is one of the most important constituents of chondrocytes, was immunostained as a chondrocyte marker according to the method of Khoshfetrat et al. (2009). Briefly, specimens were pretreated with 5,000 units/mL hyaluronidase (Calbiochem, Merck Millipore, Billerica , MA, USA) in Tris-buffered saline (TBS, Dako Cytomation, Carpinteria, CA, USA), TBS containing 1\% sodium borohyde (Sigma-Aldrich, St. Louis, MO, USA), and blocking solution (Block Ace, Dainippon Pharmaceuticals, Tokyo, Japan). A primary antibody solution containing $1 \%$ primary antibody for collagen type II (Anti-hCL(II), Daiichi Finechemicals, Toyama, Japan) and $0.1 \%$ TritonX-100, and a secondary antibody conjugated with fluorescent reagent (Alexa Fluor 568 goat anti-mouse IgG, Molecular Probes, Life Technologies Co., Carlsbad, CA, USA) were used for immunostaining.

\subsection{Optical Setup}

The SHG microscope we used consisted of an inverted confocal laser scanning microscope (DESITAL ECLIPSE C1, Nikon, Tokyo, Japan) equipped with an objective lens (20×, Plan Apo, NA = 0.75; Nikon, Tokyo, Japan) and a mode-locked Ti:sapphire laser (wavelength $\lambda=800 \mathrm{~nm}$; Chameleon, Coherent Inc., Santa Clara, CA, USA) as shown in Fig. 1. Back-scattered SHG light $(\lambda=400 \mathrm{~nm})$ from the sample was separated from the incident light with a dichroic mirror (400 nm, long pass) placed below the objective and was detected with a photomultiplier tube (H7826, 


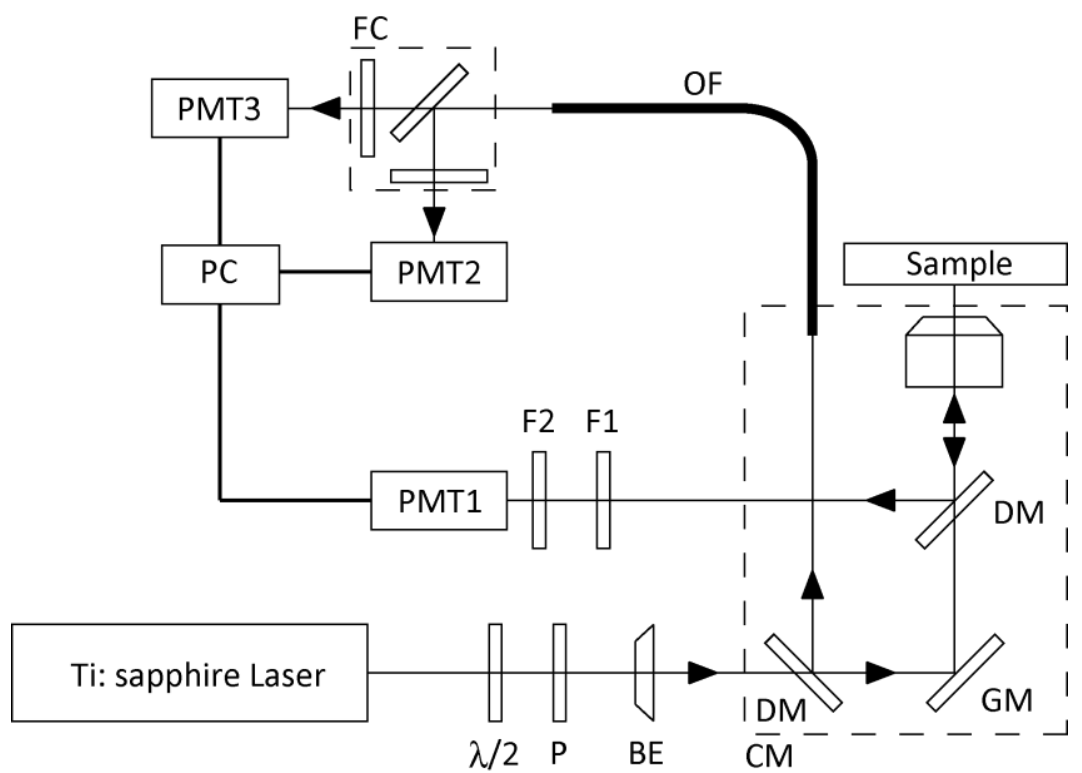

Fig. 1 Schematic view of optical setup. $\lambda / 2$ : half wave plate, P: polarizer, BE: beam expander, CM: confocal microscope, DM: dichroic mirror, GM: galvano mirror, F1: band pass filter, F2: blue pass filter, OF: optical fiber, FC: filter cube, PMT: photomultiplier tube. PMT1, 2, and 3 detects SHG, green and red fluorescence, respectively.

Hamamatsu Photonics, Hamamatsu, Japan) after passing through a band pass filter (FF01-377/50, Semrock, Lake Forest, IL, USA) and a blue pass filter (BG-39, Schott, Mainz, Germany). This microscope acquired SHG and fluorescence images simultaneously. The fluorescence was separated to green and red with a filter cube (G-2A, Nikon, Tokyo, Japan).

\subsection{Image Acquisition}

Specimens were placed between a pair of cover slips with a small amount of D-PBS to prevent displacement and drying. In order to observe the region close to the surface of the gel, the sample was oriented with this surface towards the cover slip closest to the objective lens (Fig. 2). Fluorescence and SHG images at each depth position were averaged five times in order to increase the $\mathrm{S} / \mathrm{N}$ ratio, and $\mathrm{z}$-axis sequences of 101 images were obtained in $3 \mu \mathrm{m}$ steps from the surface to a depth of $300 \mu \mathrm{m}$. The acquired images were analyzed using image analysis software (Image J, National Institute of Health, USA).

Observe the top surface of the piece

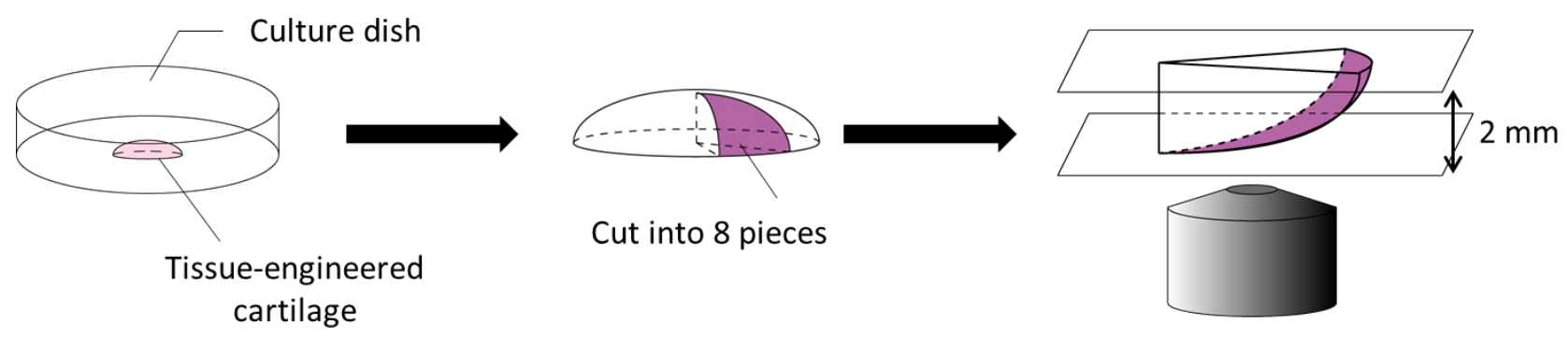

Fig. 2 Harvesting and placement of samples for observation. 


\section{Results and Discussion}

We observed tissue-engineered cartilage by SHG microscopy at 3, 8, and 15 days after seeding. In the case of the low seeding density (Fig. 3), cell number counted in $0.248 \mu \mathrm{L}$ of gel was 73 at day 3 and 249 at day 15 , and about $82 \%$ of cells showed elongated shape at day 15. The result corresponded to the previous study (Khoshfetrat et al., 2009), which showed that low-density seeding induced chondrocytes to migrate, and that such the culture showed low gene expression of collagen type II production while the gene expressions of collagen I production and migration were low. The SHG image did not change appreciably during the culture period, even in the region where an aggregation of dedifferentiated cells existed. It was because SHG occurs only in the focal spot, and only some part of stretched cells existed in the scanned plane, resulting that such kind of aggregations were not detectable as cell-shape pattern. On the other hand, with the high seeding density (Fig. 4), cell number counted in $0.248 \mu \mathrm{L}$ of gel was 73 at day 3 and 249 at day 15. All cells showed round shape at day 15, and such the cells formed aggregations. This result also corresponded to the study by Khoshfetrat et al. (2009), which showed such the culture kept chondrocyte phenotypes during the culture time. The aggregations of round cells were observed as spherical dark spaces in the SHG image (arrows in Fig. 4c). These dark spaces appeared at around 7 days after seeding, and the number of spaces increased with increasing culture time. These spaces were obviously distinguished from dark areas caused by spatial nonuniformities in the initial collagen gel. The results indicate that the spherical dark space represents the existence of an aggregation of cells which shows chondrocyte phenotypes, and it can be an evaluation factor of tissue-engineered cartilage.
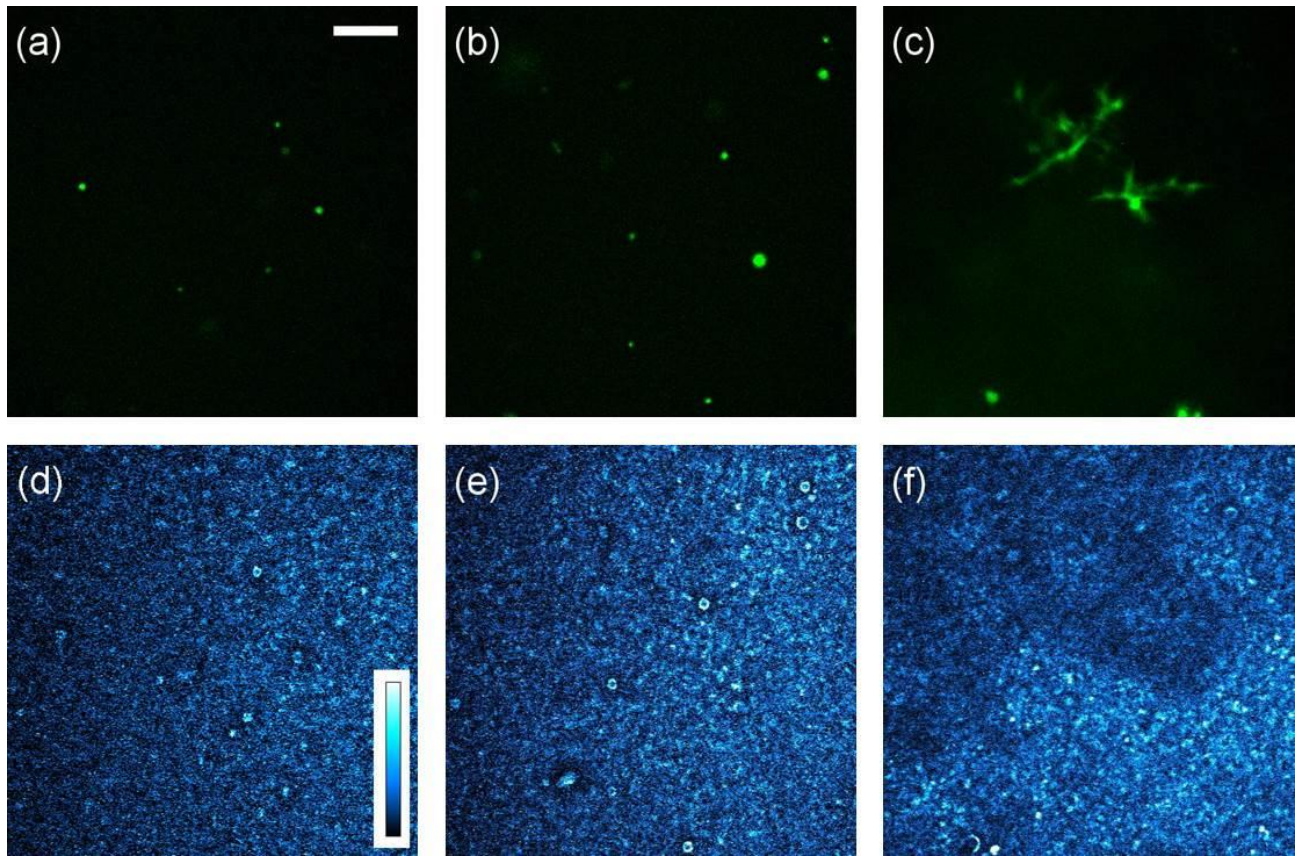

Fig. 3 Representative fluorescence $(\mathrm{a}-\mathrm{c})$ and SHG images $(\mathrm{d}-\mathrm{e})$ of tissue-engineered cartilage at low cell seeding density (2 $\times 10^{5}$ cells $/ \mathrm{mL}$ ). SHG images are shown with the range between the maximum and the minimum intensity in each image to enhance the contrast. Images were acquired at 3 days (a, d), 8 days (b, e), and 15 days (c, f) after seeding. Scale bar $=100$ 

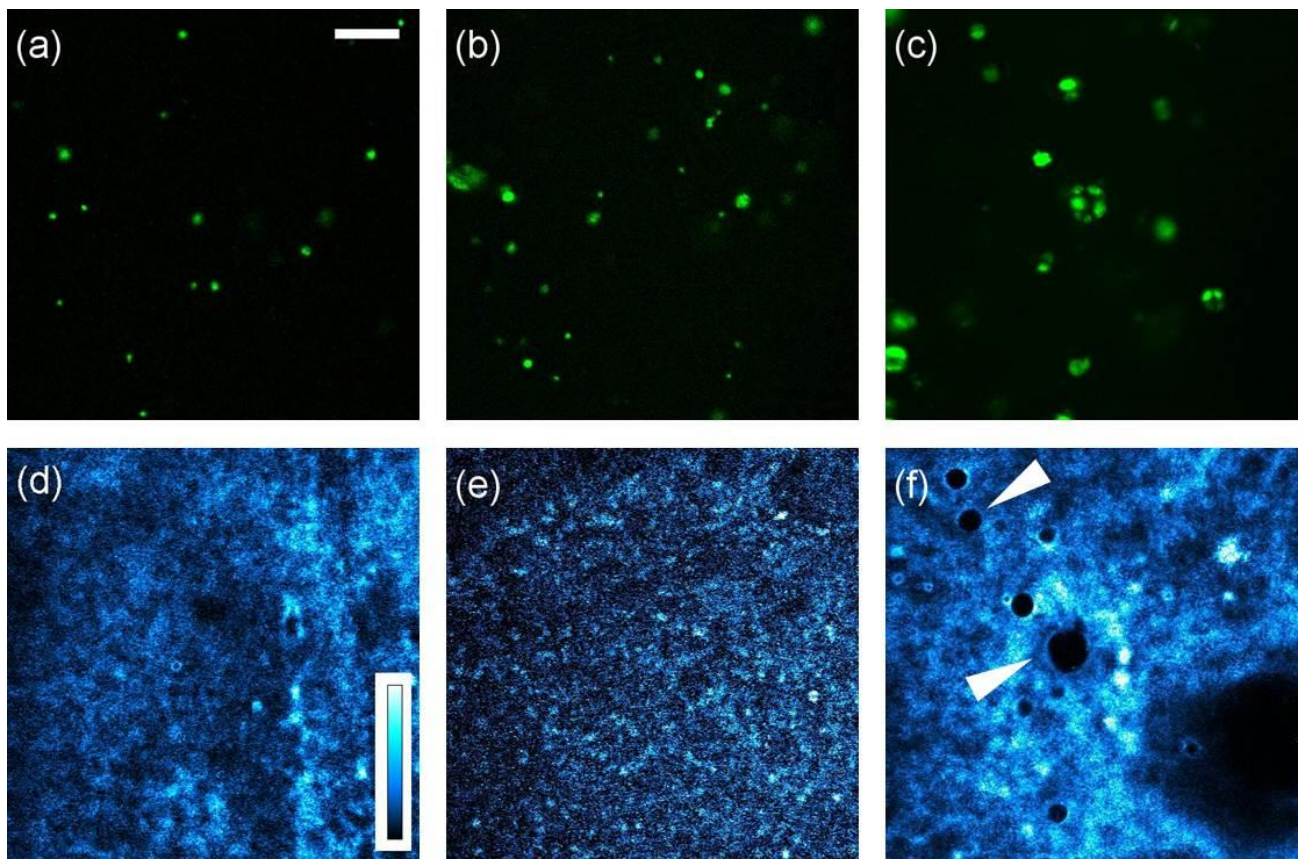

Fig. 4 Representative fluorescence $(\mathrm{a}-\mathrm{c})$ and SHG images $(\mathrm{d}-\mathrm{e})$ of tissue-engineered cartilage at high cell seeding density $\left(2 \times 10^{6}\right.$ cells $\left./ \mathrm{mL}\right)$. SHG images are shown with the range between the maximum and the minimum intensity in each image to enhance the contrast. Images were acquired at 3 days $(\mathrm{a}, \mathrm{d}), 8$ days $(\mathrm{b}, \mathrm{e})$, and 15 days $(\mathrm{c}, \mathrm{f})$ after seeding. Scale bar $=$ $100 \mu \mathrm{m}$.
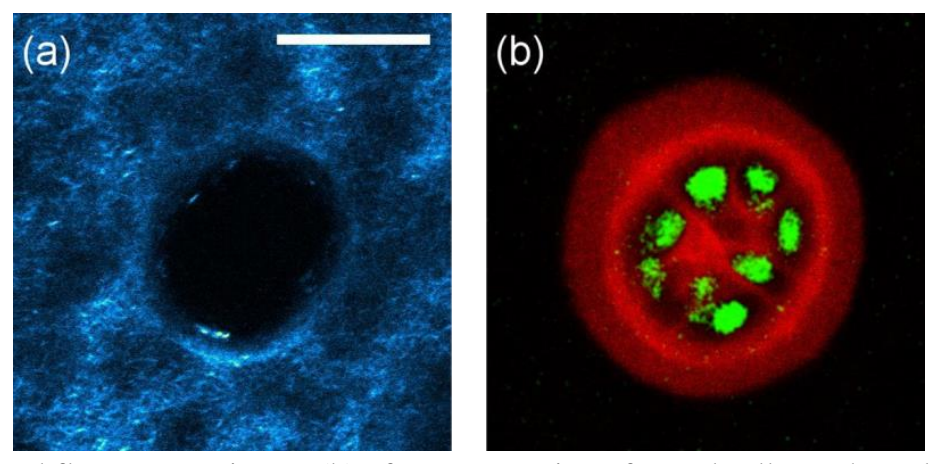

Fig. 5 SHG image (a) and fluorescence image (b) of an aggregation of round cells. The red colored regions indicate immunostained type II collagen, and the green colored regions indicate cytoplasm stained with calcein AM. Scale bar $=50$

$\mu \mathrm{m}$.

To determine the composition of the matrix at the spherical spaces in the SHG images, we immunostained the type II collagen and stained the cytoplasm. As shown in Fig. 5, the space was filled with an aggregation of round cells and type II collagen, which mainly existed in the form of a shell around the cell aggregation. We assume that the chondrocytes decomposed the environmental type I collagen gel to make room for proliferation, divided a few times, and then produced type II collagen. Type II collagen is one of the most important matrix constituents produced by chondrocytes, along with glycosaminoglycan. It is well known that all types of collagen molecule have a triple helix shape which is directional and non-centrosymmetric, and such a shape is essential for SHG. In addition, both type I and II collagens have hierarchal fibrous structures that amplify the SHG signal (Deng, et al., 2003). However, according to our results, the SHG signal emitted from the type II collagen was not detectable. This result may have been mainly due to the following two reasons. First, the SHG light intensity from type I collagen is much stronger than that from type II collagen because the emission efficiency of SHG light from type II collagen is lower than that from type I collagen on account of the difference in fibril diameter. Type II collagen molecules synthesized by chondrocytes construct thin fibrils in the presence of type XI collagen (Holmes and Kadler, 2006), whereas the fibrils of type I collagen are thick. Second, the type I collagen we used as a scaffold in the experiment was much denser than 
the type II collagen produced by the cells. Thus, presumably, the SHG intensity around the cell aggregations decreased as the type I collagen decomposed.

To verify the numbers of cells contained in spherical dark spaces, the equatorial planes of the dark spaces were selected manually from a z-series of SHG images, and the dimensions of the spaces at the planes were calculated with Image $\mathbf{J}$ software. We calculated the volumes of the dark spaces from the areas of the equatorial planes, assuming that the spaces were spheres. As shown in Fig.6, there was a strong correlation between the number of cells contained in an aggregation and the volume of the space, with a correlation coefficient of 0.86 at 15 days after seeding. This result indicates that we can non-invasively determine the number of matrix-producing chondrocytes based on the volume of the dark spaces in the SHG images. This suggests that the maturity of tissue-engineered cartilage can be estimated by monitoring the number and the volume of dark spaces in SHG images. For example, if there are no dark spaces in the SHG images at 15 days after seeding, no further cell growth can be expected. Further experiments with different culture conditions, such as different $\mathrm{pH}$ values and different growth factor concentrations, will allow us to completely understand the maturation process of chondrocytes, and based on that, we will be able to evaluate the quality sufficiently. Since the methods normally used for evaluating the quality of tissue-engineered cartilage are destructive, evaluation can be performed only once in the whole culture period, and consequently the tracking record of the culture process cannot be utilized. In contrast, quality evaluation by SHG microscopy enables us to utilize the tracking record since the method is non-destructive.

The area that can be observed with SHG microscopy has a depth limitation of $300 \mu \mathrm{m}$ from the surface because the collagen gel used in tissue-engineered cartilage is quite dense, resulting in low optical transmittance. However, such a limitation can be ignored when evaluating the quality of tissue-engineered cartilage because cells exist mostly near the surface of the gel where the oxygen concentration is sufficient for cell growth (Kino-oka et al., 2008). If it is necessary to obtain morphological information at locations deeper than $300 \mu \mathrm{m}$ inside the collagen gel, we can use longer wavelength laser light (Chu et al., 2001, Yasui et al., 2009).

In conclusion, SHG microscopy noninvasively provides us the number of matrix-producing cells. Consequently, it can be used for monitoring the culture process of tissue-engineered cartilage, as well as for quality evaluation. Using SHG microscopy to monitor the culture process is also effective for optimizing the manufacturing process.

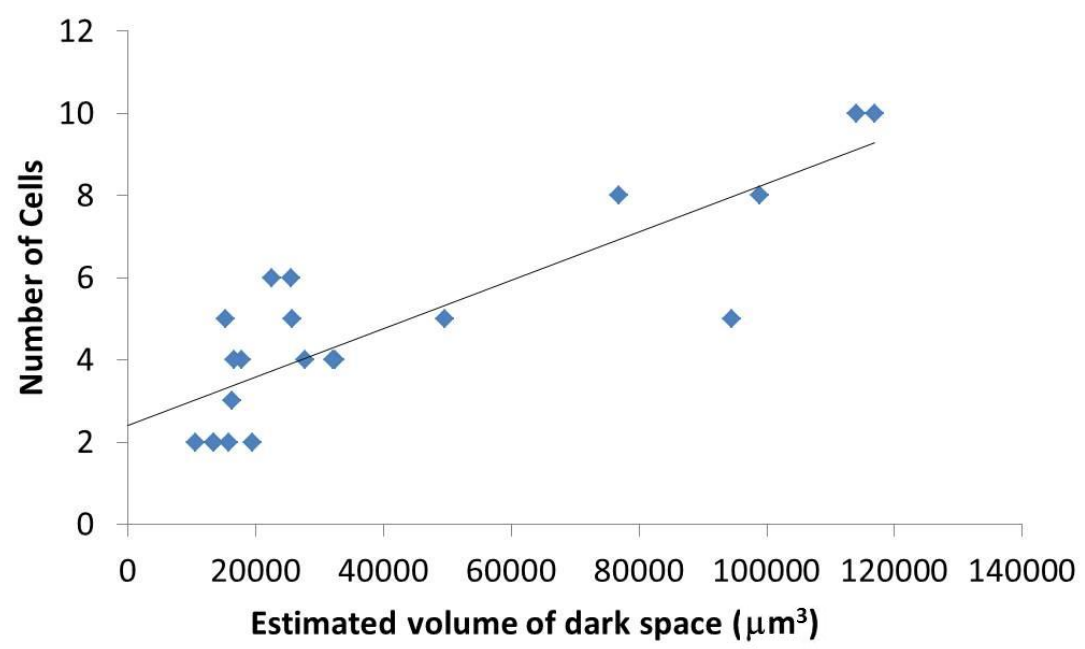

Fig. 6 Relationship between the number of constituent cells in aggregations and the estimated volume of dark spaces in SHG images. The black line represents the linear approximation.

\section{Acknowledgments}

This work was supported by Grants-in-Aid for Scientific Research from the Ministry of Education, Culture, Sports, Science, and Technology of Japan. We are grateful to Masrina Mohd Nadzir and Ali Baradar Khoshfetrat of Osaka University for technical support and fruitful discussions. 


\section{References}

Ahmed, T. A. E. and Hincke, M. T., Strategies for articular cartilage lesion repair and functional restoration, Tissue Engineering Part B: Reviews, Vol. 16, No. 3 (2010), pp. 305-329.

Chu, S. W., Chen, I. H., Liu, T. M., Chen, P. C., Sun, C. K. and Lin, B. L., Multimodal nonlinear spectral microscopy based on a femtosecond Cr:forsterite laser, Optics Letters, Vol. 26, No. 23 (2001), pp. 1909-1911.

Deng, Y., Lin, X. S., Zheng, Z., Deng, J. G., Chen, J. C., Ma, H. and Chen, G. Q., Poly(hydroxybutyrate-co-hydroxyhexanoate) promoted production of extracellular matrix of articular cartilage chondrocytes in vitro, Biomaterials, Vol. 24, No. 23 (2003), pp. 4273-4281.

Freund, I., Deutsch, M. and Sprecher, A., Connective-tissue polarity - Optical second-harmonic microscopy, crossed-beam summation, and small-angle scattering in rat-tail tendon, Biophysical Journal, Vol. 50, No. 4 (1986), pp. 693-712.

Holmes, D. F. and Kadler, K. E., The 10+4 microfibril structure of thin cartilage fibrils, Proceedings of the National Academy of Sciences of the United States of America, Vol. 103, No. 46 (2006), pp. 17249-17254.

Khoshfetrat, A. B., Kino-Oka, M., Takezawa, Y., Yamamoto, T., Sugawara, K. and Taya, M., Seeding density modulates migration and morphology of rabbit chondrocytes cultured in collagen gels, Biotechnology and Bioengineering, Vol. 102, No. 1 (2009), pp. 294-302.

Kino-oka, M., Maeda, Y., Sato, Y., Khoshfetrat, A. B., Yamamoto, T., Sugawara, K. and Taya, M., Characterization of spatial growth and distribution of chondrocyte cells embedded in collagen gels through a stereoscopic cell imaging system, Biotechnology and Bioengineering, Vol. 99, No. 5 (2008), pp. 1230-1240.

Ochi, M., Uchio, Y., Kawasaki, K., Wakitani, S. and Iwasa, J., Transplantation of cartilage-like tissue made by tissue engineering in the treatment of cartilage defects of the knee, The Journal of Bone and Joint Surgery, Vol. 84B, No. 4 (2002), pp. 571-578.

Yamamoto, T., Katoh, M., Fukushima, R., Kurushima, T. and Ochi, M., Effect of glycosaminoglycan production on hardness of cultured cartilage fabricated by the collagen-gel embedding method, Tissue Engineering, Vol. 8, No. 1 (2002), pp. 119-129.

Yasui, T., Takahashi, Y., Ito, M., Fukushima, S. and Araki, T., Ex vivo and in vivo second-harmonic-generation imaging of dermal collagen fiber in skin: comparison of imaging characteristics between mode-locked Cr:forsterite and Ti:sapphire lasers, Applied Optics, Vol. 48, No. 10 (2009), pp.D88-D95. 